\title{
Qualidade microbiológica do leite humano pasteurizado de um Banco de Leite Paulista
}

Microbiological quality of pasteurized human milk from a Milk Bank of São Paulo Calidad microbiológica de la leche humana pasteurizada de un banco de leche paulista

Claudete de Oliveira ${ }^{1}$ (D https://orcid.org/0000-0002-7385-5371 Luís Carlos Lopes-Júnior ${ }^{2}$ id https://orcid.org/0000-0002-2424-6510 Cristina Paiva de Sousa1 ic https://orcid.org/0000-0001-7151-5085

Como citar: Oliveira C, Lopes-Júnior LC, Sousa CP. Qualidade microbiológica do leite humano pasteurizado de um Banco de Leite Paulista. Acta Paul Enferm. 2022;35:eAPE00771.

DOI

http://dx.doi.org/10.37689/acta-ape/2022A000771

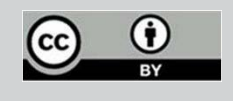

Descritores

Bancos de Leite; Controle de qualidade; Leite humano; Saúde pública; Técnicas microbiológicas

Keywords

Microbiological Techniques; Milk banks; Milk, human; Public health; Quality control

Descriptores

Bancos de leche; Control de galidad; Leche humana; Salud pública; Técnicas microbiológicas

Submetido 7 de Abril de 2020

Aceito

28 de Março de 2021

Autor correspondente

Claudete de Oliveira.

E-mail: claudete@ufscar.br

Editor Associado (Avaliação pelos pares): Adriana Amorim Francisco

(https://orcid.org/0000-0003-4705-6987)

Escola Paulista de Enfermagem, Universidade Federal de São Paulo, São Paulo, SP, Brasil

\section{Resumo}

Objetivo: Avaliar a qualidade microbiológica do leite humano pasteurizado proveniente de um Banco de Leite Humano do Estado de São Paulo.

Métodos: Estudo descritivo conduzido com 29 amostras de leite humano ordenhado pasteurizado (LHOP) obtidas entre julho de 2015 a março de 2016 por meio da avaliação dos registros da acidez titulável bem como da quantificação da microbiota heterotrófica (mesófilos, psicrófilos, termófilos), coliformes totais e termotolerantes, fungos filamentosos e leveduriformes e Staphylococcus spp. Realizou-se a avaliação dos parâmetros físico-químicos por meio do potencial hidrogeniônico-pH, teor energético-K e acidez Dornic-ºD. Análises estatísticas descritivas e bivariadas foram conduzidas.

Resultados: Evidenciou-se nas amostras a presença de psicrófilos (17,24\%), termófilos (27,59\%), mesófilos $(55,17 \%)$, fungos filamentosos e leveduriformes $(41,38 \%)$ e ausência de Staphylococcus spp. Detectou-se a presença de $82,76 \%$ de coliformes no teste presuntivo. Já no teste confirmativo VB constatou-se a presença de $54,16 \%$ de coliformes totais e no teste EC $33,33 \%$ de coliformes termotolerantes. Os valores de $\mathrm{pH}$ e de K não apresentaram oscilações, enquanto que, na expressão da acidez entre $3^{\circ}$ a $15^{\circ} \mathrm{D}$ detectou-se crescimento microbiano. 0 microrganismo mesófilo, apresentou correlação positiva com variável da acidez Dornic $(r=0.44 ; p=0.01)$.

Conclusão: A partir da avaliação da qualidade microbiológica das amostras de LHOP descartado e consideradas impróprias para consumo no referido BLH, especificamente com relação aos indicadores microbiológicos das condições de higiene, sugere que a inviabilidade das amostras possam estar associadas às boas práticas de manipulação do alimento.

\section{Abstract}

Objective: To assess the microbiological quality of pasteurized human milk from a Human Milk Bank in the State of São Paulo.

Methods: This is a descriptive study conducted with 29 pasteurized expressed human milk (PEHM) samples obtained between July 2015 and March 2016 by assessing titratable acidity records as well as quantifying heterotrophic microbiota (mesophiles, psychrophiles, thermophiles), total and thermotolerant coliforms, filamentous and yeast-like fungi and Staphylococcus spp. The physical-chemical parameters were assessed via hydrogen-pH potential, K-energy content and Dornic- ${ }^{-} \mathrm{D}$ acidity. Descriptive and bivariate statistical analyzes were conducted.

Results: The presence of psychrophiles (17.24\%), thermophiles (27.59\%), mesophiles (55.17\%), filamentous and yeast-like fungi (41.38\%) and absence of Staphylococcus spp were evidenced in the sample. The 
presence of $82.76 \%$ of coliforms was detected in the presumptive test. In the confirmatory VB test, the presence of $54.16 \%$ of total coliforms was found and, in the EC test, we verified $33.33 \%$ of thermotolerant coliforms. The $\mathrm{pH}$ and $\mathrm{K}$ values did not show oscillations, whereas, in the expression of acidity between $3^{\circ}$ and $15^{\circ} \mathrm{D}$, microbial growth was detected. The mesophilic microorganism showed a positive correlation with the Dornic acidity variable $(r=0.44 ; p=0.01)$. Conclusion: Based on the microbiological quality assessment of the HMB samples discarded and considered unfit for consumption in the HMB, specifically regarding the microbiological indicators of hygiene conditions, it suggests that the infeasibility of the samples may be associated with good food handling practices.

\section{Resumen}

Objetivo: Evaluar la calidad microbiológica de la leche humana pasteurizada proveniente de un banco de leche humana del estado de São Paulo.

Métodos: Estudio descriptivo realizado con 29 muestras de leche humana ordeñada pasteurizada (LHOP) obtenidas entre julio de 2015 y marzo de 2016 por medio de la evaluación de los registros de acidez titulable, así como de la cuantificación de la microbiota heterótrofa (mesófilos, psicrófilos, termófilos), coliformes totales y termotolerantes, hongos filamentosos y levaduriformes y Staphylococcus spp. Se realizó la evaluación de los parámetros físico-químicos mediante el potencial de hidrógeno $(\mathrm{pH})$, valor energético $(\mathrm{K})$ y acidez Dornic- ${ }^{\circ} \mathrm{D}$. Se llevaron a cabo análisis descriptivos y bivariados.

Resultados: Se observó en las muestras la presencia de psicrófilos (17,24\%), termófilos (27,59\%), mesófilos $(55,17 \%)$, hongos filamentosos y levaduriformes (41,38 \%) y ausencia de Staphylococcus spp. Se detectó la presencia del 82,76 \% de coliformes en la prueba presuntiva. Por otro lado, en la prueba confirmativa VB se confirmó la presencia del 54,16\% de coliformes totales, y en la prueba EC se verificó el 33,33 \% de coliformes termotolerantes. Los valores de $\mathrm{pH}$ y de $\mathrm{K}$ no presentaron oscilaciones, mientras que se detectó crecimiento microbiano en la expresión de la acidez entre 3 y $15^{\circ} \mathrm{D}$. El microrganismo mesófilo presentó correlación positiva con variable de la acidez Dornic $(r=0.44 ; p=0.01)$.

Conclusión: A partir de la evaluación de calidad microbiológica de las muestras de LHOP descartadas y consideradas inapropiadas para consumo en el BLH mencionado, especialmente respecto a los indicadores microbiológicos de las condiciones de higiene, se sugiere que la inviabilidad de las muestras pueda estar asociada con las buenas prácticas de manipulación del alimento.

\section{Introdução}

A literatura científica é bastante consistente ao ratificar o Leite Humano (LH) como sendo a principal fonte alimentar em recém-nascidos e lactentes. ${ }^{(1-4)}$ Dentre os benefícios desta prática destaca-se o aumento da sobrevivência, por meio da promoção da saúde e do desenvolvimento infantil, além dos benefícios biológicos e psicossociais para o desenvolvimento do bebê. ${ }^{(1,5-7)}$ No LH, além de anticorpos, encontram-se outros fatores com atividade antimicrobiana e imunomoduladora, como enzimas, citocinas, componentes do sistema complemento, oligossacarídeos, nucleotídeos, lipídeos e hormônios, que contribuem para a imunidade e maturação do sistema imunológico do neonato. ${ }^{(8-11)}$

Estudiosos da área de aleitamento materno e LH apontam que em situaçóes de vulnerabilidades incluindo a prematuridade, internaçóes recorrentes dos recém-nascidos em unidades neonatais, bem como doenças da mãe, ou baixa produção láctea, podem gerar dificuldades no estabelecimento e na manutençáo do aleitamento materno. ${ }^{(7,12,13)}$ Nessas situaçóes, o uso do LH doado se transpóe como uma eficiente opção para nutrição dos recém-nascidos em condições especiais ${ }^{(2,5)}$ e uma forma de manutenção da produção de leite pelas nutrizes doadoras. ${ }^{(5,14)}$ Recente revisão sistemática que objetivou iden- tificar as atividades dos Bancos de Leite Humano (BLH) evidenciou que as respectivas açóes neles desenvolvidas repercutem positivamente na promoção da saúde materno infantil, representando uma estratégia importante de promoção ao aleitamento e de apoio a amamentação dos bebês que não podem mamar diretamente no peito. ${ }^{(15)}$ Os autores também apontaram que pesquisas sobre os BLH como promotores da saúde materno infantil são escassas e mais estudos são necessários para subsidiar estratégias de saúde pública em prol da amamentação. ${ }^{(15)}$

Para tanto, a existência e a operacionalização dos BLH enquanto serviço especializado é crucial para desencadear açôes de promoção, proteção e apoio ao aleitamento materno bem como pela execuçáo de atividades de coleta do LH, processamento, controle de qualidade e distribuição. ${ }^{(16,17)}$ Nesse sentido, o processo da qualidade do leite humano ordenhado (LHO) abrange desde a ordenha até sua administração e é resultante das condiçóes higiênico sanitárias adequadas. Tal processo é definido por intermédio da avaliação conjunta de múltiplos parâmetros incluindo as características nutricionais, imunológicas, químicas e microbiológicas, a fim de proporcionar a verificação da segurança do produto final. ${ }^{(16,18)}$

As etapas que envolvem os procedimentos para a coleta de LHO evidenciam riscos de contaminação e, portanto, necessitam ser criteriosamente mo- 
nitoradas. ${ }^{(16,19-23)}$ É de responsabilidade do BLH o fornecimento de LHO invulnerável, inócuo, e que cumpra a sua função como alimento, já que a pasteurização inativa $95 \%$ dos agentes patogênicos. ${ }^{(16,20-23)}$ Em adição, sua qualidade é circunscrita pelo controle de inspeção em teste físico-químico e microbiológico até a liberação do produto final, sendo que as condições higiênicas sanitárias é o parâmetro aceito para viabilizar sua qualidade microbiológica. ${ }^{(20-24)}$

As causas de elevação de microrganismos no LH podem estar relacionadas às técnicas inadequadas de coleta, as condiçóes de higiene da doadora e dos utensílios e a manutenção do leite fora da cadeia de frio. $\mathrm{O}$ crescimento de bactérias no LH produz acidificação e fermentação o que pode levar a diminuição dos componentes nutricionais e imunológicos devido à utilização de nutrientes do leite pela microbiota contaminante e diminuição dos fatores imunoprotetores. $^{(24-27)}$

Uma vez que o LH é um excelente meio de cultura e não dispóe de obstáculo para a flora microbiana que está associada com a disponibilidade e a qualidade do alimento, é de suma importância para a saúde materno infantil que as boas práticas no processo de manipulação sejam asseguradas para garantir a sua qualidade. ${ }^{(17,22,24-27)}$ Dado a relevância do tema, somado a indefensabilidade materno infantil no consumo do LH distribuído por BLH, é que esta pesquisa se faz pertinente.

Embora existam dois estudos prévios, contudo, ambos abordaram a gestão do processo de trabalho das atividades assistenciais e de processamento de leite humano do BLH sob investigação nessa pesquisa com foco na melhoria da qualidade. ${ }^{(28,29)}$ No entanto, ainda permanecem desconhecidas a avaliação e a qualidade microbiológica do leite humano pasteurizado do referido BLH. Somado a isso, uma das diferenças deste estudo é que avaliamos o crescimento microbiano em diferentes tipos de temperatura, permitindo a associação da temperatura com o ambiente, armazenamento, com a degradação do leite, dentre outros parâmetros. Nesse contexto, o presente estudo objetivou avaliar a qualidade microbiológica do leite humano pasteurizado proveniente de um Banco de Leite Humano do Estado de São Paulo.

\section{Métodos}

Trata-se de estudo descritivo, feito por meio da análise e processamento das amostras de LHO obtidas em 2015/2016 no BLH vinculado à Irmandade da Santa Casa de Misericórdia de São Carlos, São Paulo, Brasil. Este estudo obteve aprovação do Comitê de Ética em Pesquisa da Universidade Federal de São Carlos - UFSCar, sob parecer $n^{\circ}: 1.8088 .916$ de 12 de maio de 2015, estando em consonância com as normas regulamentadoras contidas na Resolução 466/12 do Conselho Nacional de Saúde no que diz respeito a condução de Pesquisas com Seres Humanos. ${ }^{(30)}$ Além disso, obteve-se a aprovação junto a Comissão Interna de Ética da Irmandade da Santa Casa de Misericórdia de São Carlos - instância na qual o BLH é vinculado.

$\mathrm{O}$ processo de amostragem foi do tipo não probabilístico e por conveniência, abrangendo todas as amostras consecutivas de LH. Vinte e nove (29) amostras foram obtidas por coleta domiciliar e 01 amostra foi obtida por coleta no BLH, por doadoras cadastradas no BLH vinculado à Irmandade da Santa Casa de Misericórdia de São Carlos no período pré-estabelecido da coleta de dados do presente estudo que ocorreu entre julho de 2015 a março de 2016. Foram coletados 30 frascos, contendo $250 \mathrm{ml}$ de LHO em cada um, qualificado como impróprio para o consumo, sendo 01 amostra de LHO cru que foi descartado na fase de sujidade e 29 frascos de LHO pasteurizado, os quais foram descartados na fase de avaliação da acidez titulável expressa em graus Dornic ( $\left.{ }^{\circ} \mathrm{D}\right)$ e da qualidade microbiológica, conforme estabelecido na RDC $\mathrm{n}^{\mathrm{o}} 171$. $^{(20)}$ Ressaltase que amostra de LHO cru foi descartada devido à ausência de amostras similares para comparaçóes. Além disso, não obtivemos e nem utilizamos amostras de LHO nas fases anteriores, de análise da embalagem, de sujidades, cor e off-flavor. Dessa forma, a amostra final do presente estudo foi constituída por 29 frascos de LHO pasteurizado. As amostras foram transportadas a cadeia de frio do local de coleta no BLH até o Laboratório de Microbiologia e Biomoléculas/UFSCar em recipientes isotérmicos, respeitando as normas estabelecidas pela RDC $\mathrm{n}^{\circ}$ $171,{ }^{(20)}$ acondicionadas no freezer sob a temperatura 
de $-3^{\circ} \mathrm{C}$ para posterior processamento das análises microbiológicas em até $24 \mathrm{~h}$. A análise microbiológica foi norteada pelo método preconizado pela American Public Health Association (APHA). ${ }^{(31)}$

A análise dos parâmetros físico-químicos do potencial hidrogeniônico $(\mathrm{pH})$ do $\mathrm{LH}$ ocorreu logo após o degelo em banho-maria a $40^{\circ} \mathrm{C}$, sendo controlada para que a temperatura da água permanecesse em até $5^{\circ} \mathrm{C}$. Posteriormente, o frasco foi imerso, ficando acima da altura do nível do $\mathrm{LH}$ e realizando leves agitaçóes. Após 5 minutos, o LH foi acondicionado em um Becker $(50 \mathrm{ml})$, procedendo a mensuração do $\mathrm{pH}$ com a imersão do eletrodo no LH por 1 minuto para efetuar a leitura. Este procedimento foi repetido três vezes para cada amostra e obtivemos a média aritmética para o valor de $\mathrm{pH}$ registrado para posterior análises estatísticas. Salienta-se que os parâmetros físico-químicos do teor energético $(\mathrm{K})$ bem como os resultados da avaliação da acidez titulável ${ }^{(32)}$ foram realizados no BLH supracitado.

A detecção de microrganismos heterotróficos iniciou-se com a realização da diluição seriada da amostra de LH em solução salina peptonada. Cada amostra foi pipetada em campo de chama em diluiçôes seriadas, sendo a diluição inicial $10^{\circ}$ constituída de $10 \mathrm{ml}$ de $\mathrm{LH}$, seguida das diluiçóes $10^{-1} \mathrm{e}$ $10^{-2}$. A identificação de microrganismos heterotróficos foi realizada de acordo com o método descrito anteriormente pela APHA. ${ }^{(31)}$ Alíquotas de $1 \mathrm{ml}$ da amostra de LH e de suas diluiçôes decimais seriadas foram semeadas em duplicatas, pela técnica de inoculação em placas de petri $(90 \times 15 \mathrm{~mm})$ estéreis contendo de 10 a $15 \mathrm{ml}$ utilizando o meio de cultura Plate Count (PCA - Himedia ${ }^{\circledR}$ ). A inoculação em superfície com auxílio de uma alça de Drigalski, possibilitou que as placas fossem incubadas invertidas a $37^{\circ} \mathrm{C}$ por $24 \mathrm{~h}$ para o grupo de mesófilos; a $42,5^{\circ} \mathrm{C}$ por $24 \mathrm{~h}$ para o grupo de termófilos e a $7^{\circ} \mathrm{C}$ por 7 dias para o grupo de psicrófilos. Foram contadas as colônias e os resultados foram expressos em unidades formadoras de colônia (UFC) por $\mathrm{ml}$ de LH.

Para a análise de Staphylococcus spp. procedeu-se conforme o método proposto pela $\mathrm{APHA}^{(31)} \mathrm{com}$ a utilização do meio de cultura Agar Sal Manitol
(Kasvi $\left.^{\oplus}\right)$, no qual as placas foram incubadas e invertidas a $37^{\circ} \mathrm{C}$ por $24 \mathrm{~h}$ para verificar a presença de colônias Staphylococcus spp. Os resultados foram expressos em unidades formadoras de colônia (UFC) por ml de LH.

Para a quantificação de fungos filamentosos e leveduriformes procedeu-se novamente com o método descrito pela APHA. ${ }^{(31)}$ Assim, utilizamos o meio de cultura Agar Sabouraud Dextrose (Kasvi ${ }^{\oplus}$ ), no qual as placas foram incubadas e invertidas a $37^{\circ} \mathrm{C}$ por $24 \mathrm{~h}$, para a detecção da presença de colônias desses fungos. Os resultados também foram expressos em unidades formadoras de colônia (UFC) por $\mathrm{ml}$ de LH.

A presença de coliformes totais e coliformes termotolerantes foram determinados pela técnica do Número Mais Provável (NMP), de acordo com o método descrito em estudo prévio. ${ }^{(33)} \mathrm{O}$ teste no NMP de coliformes totais e termotolerantes foram assentados em dois testes, quais sejam, a presuntiva e a confirmativa para série de 3 tubos. O teste presuntivo foi realizado por meio de uma alíquota de $1 \mathrm{ml}$ de $\mathrm{LH}$ em um tubo contendo $9 \mathrm{ml}$ de Caldo Lauril Sulfato de Sódio (CLSS) (Kasvi ${ }^{\circledR}$ ) em diluiçôes seriadas de $10^{1}, 10^{-2}$ e $10^{-3}$ sucedendo a incubação a $37^{\circ} \mathrm{C}$ por $24 \mathrm{~h}$. O teste confirmativo foi iniciado após a verificação da inoculação dos tubos positivos por meio da formação de gás e/ou turvação do meio. Essa fase consistiu na transferência de $1 \mathrm{ml} \mathrm{da}$ diluição seriada e em triplicada para tubos contendo $9 \mathrm{ml}$ de Caldo Verde Brilhante Bile Lactose (VB) 2\% (BGBL - $\left(\mathrm{Kasvi}^{\oplus}\right)$, e para tubos contendo $9 \mathrm{ml}$ de Caldo EC (Kasvi ${ }^{\oplus}$ ), seguidos pela incubação a $37^{\circ} \mathrm{C} \mathrm{e} 42,5^{\circ} \mathrm{C}$ por $24 \mathrm{~h}$ à $48 \mathrm{~h}$. Os tubos com gás el ou turvação, nos dois testes foram registrados a fim de quantificar o $\mathrm{NMP} / \mathrm{ml}$ de $\mathrm{LH}$.

Para análise dos dados, inicialmente foram digitalizados em planilhas do Microsoft Excel $^{\mathrm{TM}}$ e após codificação, foram exportados para o programa Statistical Package for Social Sciences (SPSS Inc., Chicago, Estados Unidos), versão 20.0, para proceder-se com as análises estatísticas descritivas e bivariadas. As variáveis qualitativas ordinais e nominais foram apresentadas com base nos números absolutos e percentuais, por meio da distribuição de frequência relativa (\%) e as variáveis quantitativas discretas 
e contínuas, pela média, desvio-padrão (DP), mediana, valor mínimo (min) e valor máximo (máx). Em seguida, procedeu-se com as análises estatísticas bivariadas para investigar a correlação entre as variáveis da microbiota estudada e a acidez ${ }^{\circ}$ Dornic por meio do coeficiente de correlação de Pearson. Para todas as análises, fixou-se $\alpha=5 \%, \beta=0,20$ e Intervalo de Confiança de $95 \%$, sendo o nível de significância estabelecido para $\mathrm{p}<0,05$.

\section{Resultados}

\section{Acidez titulável}

A média dos resultados de avaliação da acidez titulável que identifica o nível de acidez expressa em graus Dornic (oD) das amostras de LHO no BLH foi de $7,8^{\circ} \mathrm{D}$, desvio padrão $(\mathrm{DP})=2,29 \circ \mathrm{D}$ (valor mínimo $3^{\circ} \mathrm{D}$, valor máximo $\left.15^{\circ} \mathrm{D}\right)$. Ademais, $76,6 \%$ das amostras de LHO $(n=23)$ apresentaram grau de titulação $\geq 8 \circ \mathrm{D}$, consideradas impróprias para consumo. No que concerne ao teor energético $(\mathrm{K})$, observou-se nas amostras de LHO do BLH uma média de $587 \mathrm{Kcal} / 1, \mathrm{DP}=77,4 \mathrm{Kcal} / \mathrm{l}$. Além disso, a média do $\mathrm{pH}$ das amostras de LHO analisadas foi de 6,8 (DP=0,22). A tabela 1 apresenta o panorama da análise físico-química da acidez Dornic, $\mathrm{pH}, \mathrm{K}$ e a quantificação de cada grupo microbiano.

\section{Cultura microbiológica}

Na tabela 2 é apresentado a quantificação de coliformes no teste presuntivo, teste confirmativo $\mathrm{VB}$ e EC respectivamente. Observou-se a ausência de coliformes em 17,24\% das amostras. Detectou-se

Tabela 1. Análise físico-química e quantificação bacteriana do Leite Humano Ordenhado Pasteurizado

\begin{tabular}{|c|c|c|c|c|c|c|c|c|c|c|c|c|c|c|c|c|c|c|c|c|c|}
\hline \multirow{3}{*}{$\begin{array}{l}\text { Amostra } \\
\mathrm{N}^{\circ}\end{array}$} & \multicolumn{3}{|c|}{$\begin{array}{l}\text { Análise físico- } \\
\text { química }\end{array}$} & \multirow{2}{*}{\multicolumn{3}{|c|}{ Psicrófilos }} & \multirow{2}{*}{\multicolumn{3}{|c|}{ Mesófilos }} & \multirow{2}{*}{\multicolumn{3}{|c|}{$\begin{array}{l}\text { UFC/ml } \\
\text { Termófilos }\end{array}$}} & & & & \multirow{2}{*}{\multicolumn{3}{|c|}{ Staphylococcus $s p$}} & & NMP $\mathrm{g} / \mathrm{ml}$ & \\
\hline & \multirow{2}{*}{${ }^{\circ} \mathrm{D}$} & \multirow{2}{*}{$\mathrm{pH}$} & \multirow[t]{2}{*}{$\mathrm{K}$} & & & & & & & & & & \multicolumn{3}{|c|}{$\begin{array}{c}\text { Fungos } \\
\text { filamentosos }\end{array}$} & & & & \multicolumn{3}{|c|}{ Coliformes } \\
\hline & & & & $10^{-1}$ & $10^{-2}$ & $10^{-3}$ & $10^{-1}$ & $10^{-2}$ & $10^{-3}$ & $10^{-1}$ & $10^{-2}$ & $10^{-3}$ & $10^{-1}$ & $10^{-2}$ & $10^{-3}$ & $10^{-1}$ & $10^{-2}$ & $10^{-3}$ & Presuntivo & VB & EC \\
\hline 1 & 8 & 6,9 & 595,2 & -- & -- & -- & -- & -- & -- & -- & -- & -- & -- & -- & -- & -- & -- & -- & -- & -- & -- \\
\hline 2 & 8 & 6,9 & 584,0 & -- & -- & -- & -- & -- & -- & -- & -- & -- & -- & -- & -- & -- & -- & -- & -- & -- & -- \\
\hline 3 & 8 & 7,0 & 586,1 & -- & -- & -- & -- & -- & -- & -- & -- & -- & -- & -- & -- & -- & -- & -- & -- & -- & -- \\
\hline 4 & 8 & 6,7 & 586,1 & -- & -- & -- & -- & -- & -- & -- & -- & -- & -- & -- & -- & -- & -- & -- & $23 \times 10^{0}$ & -- & -- \\
\hline 5 & 8 & 6,7 & 494,5 & -- & -- & -- & -- & -- & -- & -- & -- & -- & -- & -- & -- & -- & -- & -- & $9,2 \times 10^{0}$ & -- & -- \\
\hline 6 & 10 & 6,7 & 486,4 & -- & -- & -- & -- & -- & -- & -- & -- & -- & -- & -- & -- & -- & -- & -- & $23 \times 10^{0}$ & -- & -- \\
\hline 7 & 5 & 6,9 & 493,4 & 1,5 & -- & -- & -- & -- & -- & -- & -- & -- & -- & -- & -- & -- & -- & -- & $240 \times 10^{0}$ & $21 \times 10^{0}$ & -- \\
\hline 8 & 8 & 7,1 & 581,4 & 6 & 0,1 & -- & 45 & 0,2 & -- & -- & -- & -- & -- & -- & -- & -- & -- & -- & -- & -- & -- \\
\hline 10 & 8 & 7,2 & 486,4 & -- & -- & -- & -- & -- & -- & 0,5 & -- & -- & 1,5 & -- & -- & -- & -- & -- & $21 \times 10^{0}$ & -- & $21 \times 10^{0}$ \\
\hline 11 & 3 & 6,6 & 584,7 & -- & -- & -- & -- & -- & -- & -- & -- & -- & -- & -- & -- & -- & -- & -- & $21 \times 10^{0}$ & $21 \times 10^{0}$ & -- \\
\hline 12 & 5 & 6,0 & 682,9 & -- & -- & -- & -- & -- & -- & -- & -- & -- & -- & -- & -- & -- & -- & -- & $3,6 \times 10^{0}$ & $3,6 \times 10^{0}$ & -- \\
\hline 13 & 8 & 6,7 & 583,6 & -- & -- & -- & 15 & 0,5 & -- & -- & -- & -- & -- & -- & -- & -- & -- & -- & $3,6 \times 10^{0}$ & $3,6 \times 10^{0}$ & -- \\
\hline 14 & 9 & 6,7 & 593,6 & 5 & -- & -- & 20 & 0,25 & 0,05 & -- & -- & -- & 16 & -- & -- & -- & -- & -- & $3,6 \times 10^{0}$ & -- & -- \\
\hline 15 & 15 & 6,9 & 598,3 & -- & -- & -- & 30 & 0,5 & -- & -- & -- & -- & 15 & 0,15 & -- & -- & -- & -- & $160 \times 10^{0}$ & $21 \times 10^{0}$ & $23 \times 10^{0}$ \\
\hline 16 & 8 & 6,5 & 688,8 & -- & -- & -- & 4 & -- & -- & -- & -- & -- & -- & -- & -- & -- & -- & -- & $93 \times 10^{0}$ & $3,6 \times 10^{0}$ & -- \\
\hline 17 & 9 & 7,0 & 598,6 & -- & -- & -- & 45 & 0,4 & 0,05 & 9 & 0,4 & -- & 6 & 0,5 & -- & -- & -- & -- & $93 \times 10^{0}$ & -- & -- \\
\hline 18 & 8 & 6,4 & 593,6 & -- & -- & -- & -- & -- & -- & -- & -- & -- & -- & -- & -- & -- & -- & -- & -- & -- & -- \\
\hline 19 & 8 & 6,8 & 682,9 & -- & -- & -- & 4 & -- & -- & -- & -- & -- & -- & -- & -- & -- & -- & -- & $93 \times 10^{0}$ & -- & -- \\
\hline 20 & 8 & 6,8 & 682,9 & -- & -- & -- & 20 & 0,5 & -- & 2 & -- & -- & 1,5 & -- & -- & -- & -- & -- & $240 \times 10^{0}$ & $93 \times 10^{0}$ & -- \\
\hline 21 & 8 & 6,8 & 692,8 & 9 & -- & -- & 26 & 0,1 & -- & 5 & -- & -- & 4,5 & -- & -- & -- & -- & -- & $93 \times 10^{0}$ & -- & $3,6 \times 10^{0}$ \\
\hline 22 & 9 & 6,7 & 681,4 & -- & -- & -- & -- & -- & -- & -- & -- & -- & -- & -- & -- & -- & -- & -- & $150 \times 10^{0}$ & -- & $21 \times 10^{0}$ \\
\hline 23 & 8 & 6,8 & 596,7 & -- & -- & -- & -- & -- & -- & -- & -- & -- & -- & -- & -- & -- & -- & -- & $93 \times 10^{0}$ & -- & $21 \times 10^{0}$ \\
\hline 24 & 8 & 7,0 & 677,2 & -- & -- & -- & 3,5 & -- & -- & -- & -- & -- & -- & -- & -- & -- & -- & -- & $93 \times 10^{0}$ & $21 \times 10^{0}$ & -- \\
\hline 25 & 8 & 6,7 & 512,7 & 6 & -- & -- & 7,5 & 1,4 & -- & 9 & 0,1 & 0,01 & 5 & 0,2 & -- & -- & -- & -- & $15 \times 10^{0}$ & $3,6 \times 10^{0}$ & -- \\
\hline 26 & 10 & 6,9 & 666,3 & -- & -- & -- & 130 & 1,8 & -- & 16 & 1,4 & -- & 78 & 3,4 & -- & -- & -- & -- & $150 \times 10^{0}$ & $9,2 \times 10^{0}$ & $9,2 \times 10^{0}$ \\
\hline 27 & 9 & 6,7 & 583,3 & -- & -- & -- & 9 & 0,1 & -- & 3 & -- & -- & 10 & -- & -- & -- & -- & -- & $7,4 \times 10^{0}$ & $3,6 \times 10^{0}$ & -- \\
\hline 28 & 3 & 7,1 & 483,6 & -- & -- & -- & 13 & 1,3 & 0,01 & 4 & -- & -- & 2,5 & -- & -- & -- & -- & -- & $14 \times 10^{0}$ & -- & $3,6 \times 10^{0}$ \\
\hline 29 & 5 & 6,8 & 567,3 & -- & -- & -- & 78 & 0,1 & -- & -- & 24 & -- & 58 & 2,7 & -- & -- & -- & -- & $20 \times 10^{0}$ & $9,2 \times 10^{0}$ & -- \\
\hline 30 & 5 & 6,8 & 385,9 & -- & -- & -- & 60 & 0,8 & -- & -- & 7 & -- & 90 & 3,5 & -- & -- & -- & -- & $15 \times 10^{0}$ & -- & $3,6 \times 10^{0}$ \\
\hline
\end{tabular}

$\mathrm{A}^{\circ} \mathrm{D}$, acidez grau Doric; pH, potencial hidrogeniônico; $\mathrm{K}$, teor energético; UFC/ml, unidades formadoras de colônia/mililitro; NMPg/ml, número mais provável grama/mililitro; VB, meio verde brilhante; EC, meio Escherichia coli. Observação: a amostra no 9 foi descartada por ser um LHO cru 
a presença de $82,76 \%$ de coliformes no teste presuntivo. Já no teste confirmativo VB constatou-se a presença de $54,16 \%$ de coliformes totais e no teste EC verificamos 33,33\% de coliformes termotolerantes. Entretanto, em 12,5\% das amostras de LHO apresentaram teste positivo para coliformes totais e fecais.

Tabela 2. Quantificação de coliformes na fase presuntivo, confirmativo VB e EC com a acidez ${ }^{\circ} \mathrm{D}$

\begin{tabular}{|c|c|c|c|c|}
\hline \multirow{2}{*}{ Amostra } & \multirow{2}{*}{$A^{\circ} D$} & \multicolumn{3}{|c|}{ NMP g/ml - Coliformes } \\
\hline & & Presuntivo & Confirmativo VB & Confirmativo EC \\
\hline 4 & 8 & $23 \times 10^{0}$ & -- & -- \\
\hline 5 & 8 & $9,2 \times 10^{0}$ & -- & -- \\
\hline 6 & 10 & $23 \times 10^{0}$ & -- & -- \\
\hline 7 & 5 & $240 \times 10^{0}$ & $21 \times 10^{0}$ & -- \\
\hline 10 & 8 & $21 \times 10^{0}$ & -- & $21 \times 10^{0}$ \\
\hline 11 & 3 & $21 \times 10^{0}$ & $21 \times 10^{0}$ & -- \\
\hline 12 & 5 & $3,6 \times 10^{0}$ & $3,6 \times 10^{0}$ & -- \\
\hline 13 & 8 & $3,6 \times 10^{0}$ & $3,6 \times 10^{0}$ & -- \\
\hline 14 & 9 & $3,6 \times 10^{0}$ & -- & -- \\
\hline 15 & 15 & $160 \times 10^{0}$ & $21 \times 10^{0}$ & $23 \times 10^{0}$ \\
\hline 16 & 8 & $93 \times 10^{0}$ & $3,6 \times 10^{0}$ & -- \\
\hline 17 & 9 & $93 \times 10^{0}$ & -- & -- \\
\hline 19 & 8 & $93 \times 10^{0}$ & -- & -- \\
\hline 20 & 8 & $240 \times 10^{0}$ & $93 \times 10^{0}$ & -- \\
\hline 21 & 8 & $93 \times 10^{0}$ & -- & $3,6 \times 10^{0}$ \\
\hline 22 & 9 & $150 \times 10^{0}$ & -- & $21 \times 10^{0}$ \\
\hline 23 & 8 & $93 \times 10^{0}$ & -- & $21 \times 10^{0}$ \\
\hline 24 & 8 & $93 \times 10^{0}$ & $21 \times 10^{0}$ & -- \\
\hline 25 & 8 & $15 \times 10^{0}$ & $3,6 \times 10^{0}$ & -- \\
\hline 26 & 10 & $150 \times 10^{0}$ & $9,1 \times 10^{0}$ & $9,2 \times 10^{0}$ \\
\hline 27 & 9 & $7,4 \times 10^{0}$ & $3,6 \times 10^{0}$ & -- \\
\hline 28 & 3 & $14 \times 10^{0}$ & -- & $3,6 \times 10^{0}$ \\
\hline 29 & 5 & $20 \times 10^{0}$ & $9,2 \times 10^{0}$ & -- \\
\hline 30 & 5 & $15 \times 10^{0}$ & $3,6 \times 10^{0}$ & $3,6 \times 10^{0}$ \\
\hline
\end{tabular}

$\mathrm{A}^{\circ} \mathrm{D}$ - acidez grau Dornic; NMPg/mI - número mais provável grama/millilitro; $\mathrm{VB}$ - meio verde brilhante; EC - meio Escherichia coli

A distribuição do percentual dos demais microrganismos (mesófilos, psicrófilos, termófilos, fungos filamentosos e leveduriformes e Staphylococcus spp.) nas amostras de LH em função da quantificação e suas diluiçóes seriadas são mostradas na tabela 3 .

Tabela 3. Distribuição percentual da presença de microrganismos em amostras de leite humano

\begin{tabular}{|c|c|c|c|}
\hline \multirow[t]{2}{*}{ Microrganismo/Grupo } & \multicolumn{3}{|c|}{$\begin{array}{l}\text { Distribuição percentual das amostras } \\
\text { em função da quantificação e suas } \\
\text { diluições seriadas }\end{array}$} \\
\hline & $1-10^{\circ}$ & $10^{\circ}-10^{-1}$ & $10^{1} \mid-10^{-2}$ \\
\hline Psicrófilos & $17,24 \%$ & $3,45 \%$ & $0 \%$ \\
\hline Termófilos & $27,59 \%$ & $17,24 \%$ & $3,45 \%$ \\
\hline Mesófilos & $55,17 \%$ & $44,83 \%$ & $10,34 \%$ \\
\hline Fungos Filamentosos e Leveduriforme & $41,38 \%$ & $20,69 \%$ & $0 \%$ \\
\hline Staphylococcus aureus & $0 \%$ & $0 \%$ & $0 \%$ \\
\hline
\end{tabular}

O microrganismo mesófilo apresentou moderada correlaçáo positiva com a variável da acidez Dornic $(\mathrm{r}=0.44 ; \mathrm{p}=0.01)$ (Figura 1$)$. O teste da correlação linear de Pearson na presente amostra não demonstrou correlaçóes estatisticamente significativas entre os demais grupos de microrganismos e a acidez Dornic $(\mathrm{p}>0,05)$.

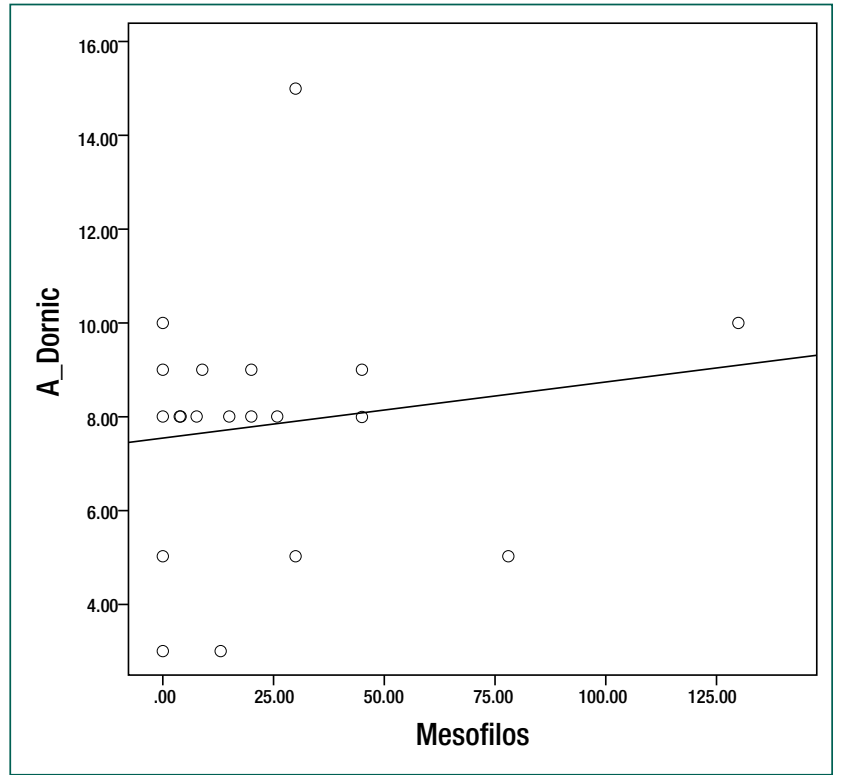

Figura 1. Correlação Linear da acidez ${ }^{\circ}$ Dornic e mesófilos $(n=30)$

\section{Discussão}

Evidenciou-se neste estudo que as amostras de leite humano ordenhado pasteurizado (LHOP) eram compostas por uma oscilaçáo de 3 a $15^{\circ}$ de acidez Dornic, sendo que 20,7\% delas apresentaram a acidez entre 3 e $5^{\circ} \mathrm{D} ; 55,2 \%$ revelaram uma acidez de $8^{\circ} \mathrm{D}$ e $24,1 \%$ acidez entre 9 e $15^{\circ} \mathrm{D}$. Em condiçôes normais a acidez Dornic no LHOP tem uma variação de $2^{\circ}$ a $7 \circ \mathrm{D}$, sendo que as amostras que apresentam acidez $\geq 8^{\circ} \mathrm{D}$ são consideradas impróprias para o consumo. Ademais, sabe-se que o descarte dessas amostras ocorre entre a fase pasteurização e a análise microbiológica, ou seja, a pasteurização tem como finalidade inativar os agentes microbianos e acidez Dornic é um parâmetro físico e indicador da qualidade higiênico sanitário do LHO. ${ }^{(12,16,20,32)}$ Para este estudo o aumento da acidez Dornic está em linha com os achados de estudos prévios $^{(32,34)}$ pois, a elevação da temperatura após 
o degelo para o processo de pasteurização interage como modificador da acidez titulável, característica observada em $13,79 \%$ de nossas amostras, as quais identificaram a acidez $8^{\circ} \mathrm{D}$ e ausência microbiana após a análise microbiológica. Nossos achados também corroboram com outro estudo que durante o armazenamento de LHO ocorreu oxidação lipídica, elevando a acidez, ${ }^{(25)}$ e 86,20\% das amostras apresentaram inviabilidade devido a presença de microrganismos. Outro estudo também demonstrou que a elevaçáo da acidez Dornic é ocasionada pelo ácido lático degradado da lactose por meio da ação microbiana. ${ }^{(19)}$

Em nosso estudo obtivemos um valor energético $(\mathrm{K})$ médio das amostras analisadas de $587 \mathrm{Kcal} / 1$, $\mathrm{DP}=77,4 \mathrm{Kcal} / \mathrm{l}$. A variabilidade do teor energético é um conjunto de resultado das características individuais das doadoras, do período da lactação, de perdas durante o processo de estocagem e da própria amostra de leite (leite de início e/ou final da mamada). ${ }^{(19)} \mathrm{A}$ média do $\mathrm{pH}$ obtida nas amostras de LHOP analisadas foi de 6,8 e este valor corrobora aos achados de outro estudo que obteve uma variação para LHOP de 6,86 (DP=0,24). ${ }^{(34)}$ Em nossas amostras, não detectamos a diminuição de $\mathrm{pH}$ em relação à acidez elevada e mesmo com a presença microbiana, provavelmente, devido a desestabilização da caseína tornando o cálcio menos disponível, fenômeno que está relacionado com a conservação inadequada do LH após a coleta. ${ }^{(19)}$ Um outro estudo ressalta para além da qualidade higiênico sanitária, que a acidez titulável se relaciona com o armazenamento em refrigeraçáo a $4^{\circ} \mathrm{C}$ no qual ocorre reaçôes lipolíticas e proteolíticas liberando ácidos graxos e aminoácidos livres, resultando na diminuição de $\mathrm{pH}$ e elevação da acidez. ${ }^{(25)}$

Foi evidenciado em 82,76\% ( $n=24)$ das nossas amostras a presença de coliformes no teste presuntivo com o valor de $3,60^{\circ}$ e $240,00^{\circ} \mathrm{NMP} / \mathrm{ml}$. Já no teste confirmativo VB constatou-se a presença de $54,16 \%$ de coliformes totais; no teste EC verificamos 33,33\% de coliformes termotolerantes e $12,5 \%$ apresentaram teste positivo para coliformes totais e termotolerantes. Este é um fator relevante considerando que sua presença é um indicador clássico para a detecção de coliformes e indica que houve a quebra das boas prá- ticas de manipulação do LH, incluindo as técnicas inadequadas de coleta, higiene precária da doadora e dos utensílios, e manutenção do leite fora da cadeia de frio. ${ }^{(16,35)}$ Além disso, a carga microbiana inicial elevada compromete a eficiência da pasteurização. Contrariamente aos nossos resultados, um estudo obteve a presença de coliformes em 10\% ( $n=2)$ das amostras analisadas de LHOP, ${ }^{(34)}$ o que corrobora a resultados de outra pesquisa ${ }^{(35)}$ na qual a avaliação das condiçóes higiênico sanitárias de LHOP, revelou a presença de 5,6\% $(n=8)$ coliformes totais e $25,9 \%$ de coliformes termotolerantes que sugeriram a contaminação fecal. ${ }^{(24)}$

Identificamos em nosso estudo a presença dos microrganismos psicrófilos em 17,24\% e de termófilos 27,59\% nas amostras de LHOP. Um estudo mostrou a presença de psicrófilos em 36,7\% de suas amostras de LHOP e de 6,7\% de microrganismos termófilos, ${ }^{(36)}$ sugerindo contaminação secundária, com manipulação ou estocagem de refrigeração inadequada.

Nossos achados revelaram 55,17\% de mesófilos e $4 \times 10^{1}$ a $130 \times 10^{1} \mathrm{UFC} / \mathrm{ml}$. Salienta-se que a quantidade permitida de microrganismos aeróbios mesófilos é de até $1 \times 10^{2} \mathrm{UFC} / \mathrm{ml}$. ${ }^{(35)}$ Esses achados são superiores aos valores encontrados por outra pesquisa $^{(24)}$ na qual foi se identificou $48,2 \%$ de mesófilos. Diferentemente, um outro estudo ${ }^{(37)}$ evidenciou a presença de $96 \%$ desse microrganismo em suas análises, corroborando aos achados de outras três pesquisas ${ }^{(38-40)}$ as quais constataram respectivamente uma população de $96 \%$ e $98,6 \%$ de mesófilos em suas amostras. ${ }^{(39)}$ Em adição, em nosso estudo, especificamente o grupo aeróbio mesófilos, após o teste da correlação linear de Pearson, demonstrou correlação positiva com a variável da acidez Dornic $(\mathrm{r}=0.44 ; \mathrm{p}=0.01)$.

A detecção de microrganismos mesófilos em amostras de LH superior ao valor de referência evidencia contaminação ocasionada à ausência das boas práticas de manipulação. As bactérias mesófilas funcionam como indicador da qualidade sanitária do LH. ${ }^{(27,38)}$ Adicionalmente, a presença desses microrganismos está relacionada as técnicas inadequadas de coleta, higiene precária em relação a doadoras e dos utensílios bem como da manutenção do leite fora da cadeia de frio. ${ }^{(5,27,34,38,40)}$ 
Identificamos ainda, $41,38 \%$ de fungos filamentosos e leveduriformes em nossas amostras. Pesquisadores encontraram uma prevalência de fungos filamentosos e leveduriformes em 69,4\% de suas amostras. ${ }^{(41)}$ Já em outro estudo foi reportado a presença de fungos filamentosos e leveduriformes em $56 \%$ de suas amostras. ${ }^{(39)} \mathrm{A}$ incidência de fungos filamentosos e leveduriformes sugerem a transferência para as máos das doadoras e consequentemente ao LHO, fato de extrema relevância para o controle da assepsia e da manipulação adequada durante a coleta de LHO a fim de evitar contaminação. ${ }^{(33)}$

Ressalta-se que, em nosso estudo não foram encontradas crescimento microbiano de Staphylococcus spp semelhante a outros dois estudos com 20 e 14 amostra de LHOP respectivamente. ${ }^{(34,40)}$ Este microrganismo faz parte do grupo de bactérias mesófilas possuindo um crescimento ótimo a $37^{\circ} \mathrm{C}$ e na faixa entre 7 a $47,8^{\circ} \mathrm{C}$, além de apresentar um crescimento microbiano em $\mathrm{pH}$ de 4 e 9,8. Portanto, a presença desse microrganismo no LH é um indicativo de contaminante externo, proveniente de manipuladores, utensílios e equipamentos. ${ }^{(21,36,40)}$

Reconhecemos algumas limitações do presente estudo, dentre elas o tamanho pequeno da amostra que impossibilitou a condução de análises de subgrupos e análises multivariadas. Outra limitação é que a maioria dos estudos prévios na literatura reflete sobre o LHO cru e o nosso estudo utilizou o LHOP, o que pode ter dificultado algumas comparaçôes. Entretanto, este estudo diferentemente dos previamente publicados, utilizou o grupo de microrganismo indicador microbiológico das condições de higiene nos alimentos por meio da variação da temperatura, não optando em classificá-lo.

\section{Conclusão}

A partir da avaliação da qualidade microbiológica das amostras de LHOP descartado e consideradas impróprias para consumo no referido BLH, especificamente com relação aos indicadores microbiológicos das condiçóes de higiene, sugere que a inviabilidade das amostras possam estar associadas às boas práticas de manipulação do alimento. Dentre elas, às técnicas inadequadas de coleta, à conservação inadequada do LHO após a coleta, e contaminação secundária, com manipulação ou estocagem de refrigeração inadequada, que podem ter sido ocasionadas tanto pelas doadoras quanto pelo próprio BLH. Espera-se com os nossos achados possam contribuir para a reflexão de gestores e profissionais de saúde, destacando a necessidade da Educação Permanente junto aos BLH a fim de melhorar as boas práticas de manipulação do LH, principalmente, em consonância com a qualidade higiênico sanitária na coleta do mesmo, uma vez que ela náo exerce monitoramento absoluto na coleta externa.

\section{Colaborações}

Oliveira C, Lopes-Júnior LC e Sousa CP contribuíram com a concepçáo do estudo, análise e interpretação dos dados, redação do artigo, revisão crítica relevante do conteúdo intelectual e aprovação da versão final ser publicada.

\section{Referências}

1. Victora CG, Bahl R, Ramos AJ, França GV, Horton S, Krasevek J, Murch S, Sankar MJ, Walker N, Rollins NC; Lancet Breastfeeding Series Group. Breastfeeding in the 21 st century: epidemiology, mechanisms, and lifelong effect. Lancet. 2016;387(10017):475-90.

2. Section on Breastfeeding. Breastfeeding and the use of human milk. Pediatrics. 2012;129(3):827-41.

3. World Health Organization (WHO). The optimal duration of exclusive breastfeeding: report of an expert consultation. Geneva: WHO; 2001.

4. DeMarchis A, Israel-Ballard K, Mansen KA, Engmann C. Establishing an integrated human milk banking approach to strengthen newborn care. J Perinatol. 2017;37(5):469-74. Review.

5. Wesolowska A, Sinkiewicz-Darol E, Barbarska 0, BernatowiczLojko U, Borszewska-Kornacka MK, van Goudoever JB. Innovative Techniques of processing human milk to preserve key components. Nutrients. 2019;11(5):1169. Review.

6. Boquien CY. Human milk: an ideal food for nutrition of preterm newborn. Front Pediatr. 2018;6:295. Review.

7. Tonon KM, Miranda A, Abrão AC, de Morais MB, Morais TB. Validation and application of a method for the simultaneous absolute quantification of 16 neutral and acidic human milk oligosaccharides by graphitized carbon liquid chromatography - electrospray ionization mass spectrometry. Food Chem. 2019;274:691-7.

8. Palmeira P, Costa-Carvalho BT, Arslanian C, Pontes GN, Nagao AT, Carneiro-Sampaio MM. Transfer of antibodies across the placenta and in breast milk from mothers on intravenous immunoglobulin. Pediatr Allergy Immunol. 2009;20(6):528-35. 
9. Beghetti I, Biagi E, Martini S, Brigidi P, Corvaglia L, Aceti A. Human milk's hidden gift: implications of the milk microbiome for preterm infants' health. Nutrients. 2019;11(12):2944. Review.

10. Ballard 0 , Morrow AL. Human milk composition: nutrients and bioactive factors. Pediatr Clin North Am. 2013;60(1):49-74. Review.

11. Peila C, Moro GE, Bertino E, Cavallarin L, Giribaldi M, Giuliani F, et al. The effect of holder pasteurization on nutrients and biologically-active components in donor human milk: a review. Nutrients. 2016;8(8):477. Review.

12. Menezes G, Lima-Cavalcanti L, Morais-Oliveira AM, Costa-Pinto RM, Steffen Abdallah V0. Evaluación de la recolección domiciliaria feita por un banco de leche humana de un hospital universitario de Brasil. Salud Publica Mex. 2014;56:245-50.

13. Carreiro JA, Francisco AA, Abrão AC, Marcacine KO, Abuchaim ES, Coca KP. Breastfeeding diffi culties: analysis of a service specialized in breastfeeding. Acta Paul Enferm. 2018;31(4):430-8.

14. Lubbe W, Oosthuizen CS, Dolman RC, Covic N. Stakeholder attitudes towards donating and utilizing donated human breastmilk. Int J Environ Res Public Health. 2019;16(10):1838.

15. Fonseca RM, Milagres LC, Franceschini SC, Henriques BD. The role of human milk banks in promoting maternal and infant health: a systematic review. Ciênc Saúde Coletiva. 2021;26(1):309-18.

16. Agência Nacional de Vigilância Sanitária (ANVISA). Banco de Leite Humano: funcionamento, prevenção e controle de riscos. Brasília (DF): ANVISA; 2008.

17. Moreno MA, Castro LS, Abrão AC, Coca KP. Food safety and quality of distribution of raw human milk from a University Hospital. Rev Nutr. 2018;31(6):547-56.

18. Vázquez-Román S, Garcia-Lara NR, Escuder-Vieco D, Chaves-Sánchez F, De la Cruz-Bertolo J, Pallas-Alonso CR. Determination of dornic acidity as a method to select donor milk in a milk bank. Breastfeed Med. 2013;8(1):99-104.

19. Rona MS, Novak FR, Portilho M, Pelissari FM, Martins AB, Matioli G. Efeito do tempo e da temperatura de estocagem nas determinações de acidez, cálcio, proteínas e lipídeos de leite de doadoras de bancos de leite humano. Rev Bras Saúde Matern Infant. 2008;8(3):257-63.

20. Brasil. Ministério da Saúde. Agência Nacional de Vigilância Sanitária. Resolução RDC $n^{0}$ 171, de 04 de setembro de 2006. Dispõe sobre 0 Regulamento Técnico para o Funcionamento de Bancos de Leite Humano [Internet]. Brasília (DF): Ministério da Saúde; 2006 [citado 2020 Mar 6]. Disponivel em: https://bvsms.saude.gov.br/bvs/saudelegis/anvisa/2006/ res0171_04_09_2006.html\#: :text=Estabelecer\%200s\%20 requisitos\%20para\%20instala\%C3\%A7\%C3\%A30,sanit\%C3\%A1ria\%20 do\%20leite\%20humano\%20ordenhado

21. Bhisikar S, Mondkar J, Manerkar S, Goel S, D'Dsouza D, Baveja S Improving quality of banked milk: utility of dornic acid test. Indian $\mathrm{J}$ Pediatr. 2018;85(4):272-5.

22. Barros M, Almeida JG, Rabuffetti A. Rede brasileira de bancos de leite humano: uma rede baseada na confiança. Rev Eletron Comun Inf Inov Saúde. 2018;12(2):125-33.

23. Sousa BR, Leite CM, Martins WS, Machado AV, Medeiros AC, Pereira $\mathrm{KE}$, et al. Aspectos qualitativos do leite humano. Infor Téc Semiárido. 2017;11(1):67-71.

24. Pontes MR, Ivasaki Y, Oliveira YS. Avaliação das condições sanitárias do leite humano pasteurizado distribuído pelo banco de leite de um hospital público do Distrito federal. Hig Aliment. 2003;17:43-49.
25. Galhardo AL, Araújo WM, Borgo LA. Acidez Dornic como parâmetro de qualidade, em bancos de leite humano. Hig Aliment. 2002;16(100):16-27.

26. Novak FR, Almeida JA, Asensi MD, Moraes BA, Rodrigues DP. Resistência antimicrobiana de coliformes isolados de leite humano ordenhado. Cad Saude Publica. 2001;17(3):713-7.

27. Borges MS, Oliveira AM, Hattori WT, Abdallah VO. Quality of human milk expressed in a human milk bank and at home. J Pediatr (Rio J). 2018;94(4):399-403.

28. Torrezan AC. Gestão do processo assistencial e de processamento de Banco de Leite Humano com foco na melhoria da qualidade [dissertação]. São Carlos: UFSCar; 2011. 79f.

29. Silveira JQ, Pereira CA. Identificação de pontos críticos de controle no banco de leite humano da Santa Casa de Misericórdia em São Carlos/ SP. Nutrição Brasil. 2011;10(3):161-8.

30. Brasil, Conselho Nacional de Saúde (CNS). Resolução 466/2012 Normas para pesquisa envolvendo seres humanos [Internet]. Brasília (DF): CNS; 2012 [citado 2020 Mar 6]. Disponível em: https://conselho. saude.gov.br/resolucoes/2012/Reso466.pdf

31. Salfinger $Y$, Tortorello ML. Compendium of methods for the microbiological examination of foods. Washington: American Public Health Association; 2015.

32. Cavalcante JL, Teles FJ, Peixoto MM, Rodrigues RC. Uso da acidez titulável no controle de qualidade do leite humano ordenhado. Food Sci Technol. 2005;25(1):103-8.

33. Novak FR, Almeida JA. Teste alternativo para detecção de coliformes em leite humano ordenhado J Pediatr (Rio J). 2002;78(3):587-91.

34. Sousa PP, Silva JA. Monitoramento da qualidade do leite humano ordenhado e distribuído em banco de leite de referência. Rev Inst Adolfo Lutz. 2010;69(1):7-14.

35. Serafini AB, André MC, Rodrigues MA, Kipnis A, Carvalho C0, Campos $\mathrm{MR}$, et al. Microbiological quality of human milk from a Brazilian milk bank. Rev Saude Publica. 2003;37(6):755-9.

36. Novak FR, Junqueira AR, Dias MS, Almeida JA. Sensorial analysis of expressed human milk and its microbial load. J Pediatr (Rio J). 2008;84(2):181-4.

37. Mesquita M, Silva A, Tavares AP, Almeida A. Contagem de bactérias mesófilas aeróbias e características físico-químicas do leite humano armazenado em embalagem de polietileno. Vigil Sanit Debate. 2016;4(3):51-6.

38. Novak FR, Cordeiro DM. The Correlation between aerobic mesoplilic microoganism counts and Dornic acidith in expressed human breastmilk. J Pediatr (Rio J). 2007;83(1):87-91.

39. Scarso IS, Valle RV, Lira BB, Teixeira EP, Fonseca YS, Arine ML, et al. Análise Físico-química e bacteriológica de leite cru e pasteurizado do Banco de Leite Humano de Sorocaba, SP. Hig Aliment. 2006;20(142):85-9.

40. Sousa C, Neves E, Lourenço L, Lucena M, Lins R. Diagnostico das condições higiênicas e microbiológicas do Banco de Leite Humano do Hospital Santa Casa de Misericórdia, na cidade de Belém, Estado do Pará. Alim Nutr. 2007;18(2):133-40.

41. Almeida VM, Nascimento AR, Chaves NP, Bezerra DC, Alves LM. Diagnóstico das condições higiênicosanitárias de um banco de leite humano na cidade de São Luís, MA, Brasil. Alim Nutr. 2012;23(1):95-99. 